Geometry $\&$ Topology Monographs

Volume 1: The Epstein birthday schrift

Pages 383-411

\title{
Hilbert's 3rd Problem and Invariants of 3-manifolds
}

\author{
Walter D NeumanN
}

\begin{abstract}
This paper is an expansion of my lecture for David Epstein's birthday, which traced a logical progression from ideas of Euclid on subdividing polygons to some recent research on invariants of hyperbolic 3manifolds. This "logical progression" makes a good story but distorts history a bit: the ultimate aims of the characters in the story were often far from 3-manifold theory.

We start in section 1 with an exposition of the current state of Hilbert's 3rd problem on scissors congruence for dimension 3 . In section 2 we explain the relevance to 3-manifold theory and use this to motivate the Bloch group via a refined "orientation sensitive" version of scissors congruence. This is not the historical motivation for it, which was to study algebraic $K-$ theory of $\mathbb{C}$. Some analogies involved in this "orientation sensitive" scissors congruence are not perfect and motivate a further refinement in section 4 . Section 5 ties together various threads and discusses some questions and conjectures.
\end{abstract}

AMS Classification 57M99; 19E99, 19F27

Keywords Scissors congruence, hyperbolic manifold, Bloch group, dilogarithm, Dehn invariant, Chern-Simons

\section{Hilbert's 3rd Problem}

It was known to Euclid that two plane polygons of the same area are related by scissors congruence: one can always cut one of them up into polygonal pieces that can be re-assembled to give the other. In the 19th century the analogous result was proved with euclidean geometry replaced by 2-dimensional hyperbolic geometry or 2-dimensional spherical geometry.

The 3rd problem in Hilbert's famous 1900 Congress address [18] posed the analogous question for 3-dimensional euclidean geometry: are two euclidean polytopes of the same volume "scissors congruent," that is, can one be cut into subpolytopes that can be re-assembled to give the other. Hilbert made clear that he expected a negative answer. 
One reason for the nineteenth century interest in this question was the interest in a sound foundation for the concepts of area and volume. By "equal area" Euclid meant scissors congruent, and the attempt in Euclid's Book XII to provide the same approach for 3-dimensional euclidean volume involved what was called an "exhaustion argument" - essentially a continuity assumption that mathematicians of the nineteenth century were uncomfortable with (by Hilbert's time mostly for aesthetic reasons).

The negative answer that Hilbert expected to his problem was provided the same year ${ }^{1}$ by Max Dehn [7]. Dehn's answer is delighfully simple in modern terms, so we describe it here in full.

Definition 1.1 Consider the free $\mathbb{Z}$-module generated by the set of congruence classes of 3-dimensional polytopes. The scissors congruence group $\mathcal{P}\left(\mathbb{E}^{3}\right)$ is the quotient of this module by the relations of scissors congruence. That is, if polytopes $P_{1}, \ldots, P_{n}$ can be glued along faces to form a polytope $P$ then we set

$$
[P]=\left[P_{1}\right]+\cdots+\left[P_{n}\right] \text { in } \mathcal{P}\left(\mathbb{E}^{3}\right) .
$$

(A polytope is a compact domain in $\mathbb{E}^{3}$ that is bounded by finitely many planar polygonal "faces.")

Volume defines a map

$$
\text { vol }: \mathcal{P}\left(\mathbb{E}^{3}\right) \rightarrow \mathbb{R}
$$

and Hilbert's problem asks ${ }^{2}$ about injectivity of this map.

Dehn defined a new invariant of scissors congrence, now called the Dehn invariant, which can be formulated as a map $\delta: \mathcal{P}\left(\mathbb{E}^{3}\right) \rightarrow \mathbb{R} \otimes \mathbb{R} / \pi \mathbb{Q}$, where the tensor product is a tensor product of $\mathbb{Z}$-modules (in this case the same as tensor product as $\mathbb{Q}$-vector spaces).

\footnotetext{
${ }^{1}$ In fact, the same answer had been given in 1896 by Bricard, although it was only fully clarified around 1980 that Bricard was answering an equivalent question — see Sah's review 85f:52014 (AMS Mathematical Reviews) of [9] for a concise exposition of this history.

${ }^{2}$ Strictly speaking this is not quite the same question since two polytopes $P_{1}$ and $P_{2}$ represent the same element of $\mathcal{P}\left(\mathbb{E}^{3}\right)$ if and only if they are stably scissors congruent rather than scissors congruent, that is, there exists a polytope $Q$ such that $P_{1}+Q$ (disjoint union) is scissors congruent to $P_{2}+Q$. But, in fact, stable scissors congruence implies scissors congruence $([47,48]$, see $[35]$ for an exposition).
} 
Definition 1.2 If $E$ is an edge of a polytope $P$ we will denote by $\ell(E)$ and $\theta(E)$ the length of $E$ and dihedral angle (in radians) at $E$. For a polytope $P$ we define the Dehn invariant $\delta(P)$ as

$$
\delta(P):=\sum_{E} \ell(E) \otimes \theta(E) \quad \in \quad \mathbb{R} \otimes(\mathbb{R} / \pi \mathbb{Q}), \quad \text { sum over all edges } E \text { of } P .
$$

We then extend this linearly to a homomorphism on $\mathcal{P}\left(\mathbb{E}^{3}\right)$.

It is an easy but instructive exercise to verify that

- $\delta$ is well-defined on $\mathcal{P}\left(\mathbb{E}^{3}\right)$, that is, it is compatible with scissors congruence;

- $\delta$ and vol are independent on $\mathcal{P}\left(\mathbb{E}^{3}\right)$ in the sense that their kernels generate $\mathcal{P}\left(\mathbb{E}^{3}\right)$ (whence $\operatorname{Im}(\delta \mid \operatorname{Ker}(\operatorname{vol}))=\operatorname{Im}(\delta)$ and $\left.\operatorname{Im}(\operatorname{vol} \mid \operatorname{Ker}(\delta))=\mathbb{R}\right)$;

- the image of $\delta$ is uncountable.

In particular, ker(vol) is not just non-trivial, but even uncountable, giving a strong answer to Hilbert's question. To give an explicit example, the regular simplex and cube of equal volume are not scissors congruent: a regular simplex has non-zero Dehn invariant, and the Dehn invariant of a cube is zero.

Of course, this answer to Hilbert's problem is really just a start. It immediately raises other questions:

- Are volume and Dehn invariant sufficient to classify polytopes up to scissors congruence?

- What about other dimensions?

- What about other geometries?

The answer to the first question is "yes." Sydler proved in 1965 that

$$
(\operatorname{vol}, \delta): \mathcal{P}\left(\mathbb{E}^{3}\right) \rightarrow \mathbb{R} \oplus(\mathbb{R} \otimes \mathbb{R} / \pi \mathbb{Q})
$$

is injective. Later Jessen $[19,20]$ simplified his difficult argument somewhat and proved an analogous result for $\mathcal{P}\left(\mathbb{E}^{4}\right)$ and the argument has been further simplified in [13]. Except for these results and the classical results for dimensions $\leq 2$ no complete answers are known. In particular, fundamental questions remain open about $\mathcal{P}\left(\mathbb{H}^{3}\right)$ and $\mathcal{P}\left(\mathbb{S}^{3}\right)$.

Note that the definition of Dehn invariant applies with no change to $\mathcal{P}\left(\mathbb{H}^{3}\right)$ and $\mathcal{P}\left(\mathbb{S}^{3}\right)$. The Dehn invariant should be thought of as an "elementary" invariant, since it is defined in terms of 1 -dimensional measure. For this reason (and other 
reasons that will become clear later) we are particularly interested in the kernel of Dehn invariant, so we will abbreviate it: for $\mathbb{X}=\mathbb{E}^{3}, \mathbb{H}^{3}, \mathbb{S}^{3}$

$$
\mathcal{D}(\mathbb{X}):=\operatorname{Ker}(\delta: \mathcal{P}(\mathbb{X}) \rightarrow \mathbb{R} \otimes \mathbb{R} / \pi \mathbb{Q})
$$

In terms of this notation Sydler's theorem that volume and Dehn invariant classify scissors congruence for $\mathbb{E}^{3}$ can be reformulated:

$$
\text { vol: } \mathcal{D}\left(\mathbb{E}^{3}\right) \rightarrow \mathbb{R} \quad \text { is injective. }
$$

It is believed that volume and Dehn invariant classify scissors congruence also for hyperbolic and spherical geometry:

Conjecture 1.3 Dehn Invariant Sufficiency vol: $\mathcal{D}\left(\mathbb{H}^{3}\right) \rightarrow \mathbb{R}$ is injective and vol: $\mathcal{D}\left(\mathbb{S}^{3}\right) \rightarrow \mathbb{R}$ is injective.

On the other hand vol: $\mathcal{D}\left(\mathbb{E}^{3}\right) \rightarrow \mathbb{R}$ is also surjective, but this results from the existence of similarity transformations in euclidean space, which do not exist in hyperbolic or spherical geometry. In fact, Dupont [8] proved:

Theorem 1.4 vol: $\mathcal{D}\left(\mathbb{H}^{3}\right) \rightarrow \mathbb{R}$ and vol: $\mathcal{D}\left(\mathbb{S}^{3}\right) \rightarrow \mathbb{R}$ have countable image.

Thus the Dehn invariant sufficiency conjecture would imply:

Conjecture 1.5 Scissors Congruence Rigidity $\mathcal{D}\left(\mathbb{H}^{3}\right)$ and $\mathcal{D}\left(\mathbb{S}^{3}\right)$ are countable.

The following collects results of Bökstedt, Brun, Dupont, Parry, Sah and Suslin ([3], [12], [36], [37]).

Theorem 1.6 $\mathcal{P}\left(\mathbb{H}^{3}\right)$ and $\mathcal{P}\left(\mathbb{S}^{3}\right)$ and their subspaces $\mathcal{D}\left(\mathbb{H}^{3}\right)$ and $\mathcal{D}\left(\mathbb{S}^{3}\right)$ are uniquely divisible groups, so they have the structure of $\mathbb{Q}$-vector spaces. As $\mathbb{Q}$ vector spaces they have infinite rank. The rigidity conjecture thus says $\mathcal{D}\left(\mathbb{H}^{3}\right)$ and $\mathcal{D}\left(\mathbb{S}^{3}\right)$ are $\mathbb{Q}$-vector spaces of countably infinite rank.

Corollary 1.7 The subgroups $\operatorname{vol}\left(\mathcal{D}\left(\mathbb{H}^{3}\right)\right)$ and $\operatorname{vol}\left(\mathcal{D}\left(\mathbb{S}^{3}\right)\right)$ of $\mathbb{R}$ are $\mathbb{Q}$-vector subspaces of countable dimension. 


\section{$1.1 \quad$ Further comments}

Many generalizations of Hilbert's problem have been considered, see eg [35] for an overview. There are generalizations of Dehn invariant to all dimensions and the analog of the Dehn invariant sufficiency conjectures have often been made in greater generality, see eg [35], [12], [16]. The particular Dehn invariant that we are discussing here is a codimension 2 Dehn invariant.

Conjecture 1.3 appears in various other guises in the literature. For example, as we shall see, the $\mathbb{H}^{3}$ case is equivalent to a conjecture about rational relations among special values of the dilogarithm function which includes as a very special case a conjecture of Milnor [22] about rational linear relations among values of the dilogarithm at roots of unity. Conventional wisdom is that even this very special case is a very difficult conjecture which is unlikely to be resolved in the forseeable future. In fact, Dehn invariant sufficiency would imply the ranks of the vector spaces of volumes in Corollary 1.7 are infinite, but at present these ranks are not even proved to be greater than 1. Even worse: although it is believed that the volumes in question are always irrational, it is not known if a single one of them is!

As we describe later, work of Bloch, Dupont, Parry, Sah, Wagoner, and Suslin connects the Dehn invariant kernels with algebraic $K$-theory of $\mathbb{C}$, and the above conjectures are then equivalent to standard conjectures in algebraic $K-$ theory. In particular, the scissors congruence rigidity conjectures for $H^{3}$ and $S^{3}$ are together equivalent to the rigidity conjecture for $K_{3}(\mathbb{C})$, which can be formulated that $K_{3}^{\text {ind }}(\mathbb{C})$ (indecomposable part of Quillen's $K_{3}$ ) is countable. This conjecture is probably much easier than the Dehn invariant sufficiency conjecture.

The conjecture about rational relations among special values of the dilogarithm has been broadly generalized to polylogarithms of all degrees by Zagier (section 10 of [46]). The connections between scissors congruence and algebraic $K-$ theory have been generalised to higher dimensions, in part conjecturally, by Goncharov [16].

We will return to some of these issues later. We also refer the reader to the very attractive exposition in [14] of these connnections in dimension 3.

I would like to acknowledge the support of the Australian Research Council for this research, as well as the the Max-Planck-Institut für Mathematik in Bonn, where much of this paper was written. 


\section{Hyperbolic 3-manifolds}

Thurston's geometrization conjecture, much of which is proven to be true, asserts that, up to a certain kind of canonical decomposition, 3-manifolds have geometric structures. These geometric structures belong to eight different geometries, but seven of these lead to manifolds that are describable in terms of surface topology and are very easily classified. The eighth geometry is hyperbolic geometry $\mathbb{H}^{3}$. Thus if one accepts the geometrization conjecture then the central issue in understanding 3-manifolds is to understand hyperbolic 3manifolds.

Suppose therefore that $M=\mathbb{H}^{3} / \Gamma$ is a hyperbolic 3-manifold. We will always assume $M$ is oriented and for the moment we will also assume $M$ is compact, though we will be able to relax this assumption later. We can subdivide $M$ into small geodesic tetrahedra, and then the sum of these tetrahedra represents a class $\beta_{0}(M) \in \mathcal{P}\left(\mathbb{H}^{3}\right)$ which is an invariant of $M$. We call this the scissors congruence class of $M$.

Note that when we apply the Dehn invariant to $\beta_{0}(M)$ the contributions coming from each edge $E$ of the triangulation sum to $\ell(E) \otimes 2 \pi$ which is zero in $\mathbb{R} \otimes \mathbb{R} / \pi \mathbb{Q}$. Thus

Proposition 2.1 The scissors congruence class $\beta_{0}(M)$ lies in $\mathcal{D}\left(\mathbb{H}^{3}\right)$.

How useful is this invariant of $M$ ? We can immediately see that it is non-trivial, since at least it detects volume of $M$ :

$$
\operatorname{vol}(M)=\operatorname{vol}\left(\beta_{0}(M)\right) .
$$

Now it was suggested by Thurston in [42] that the volume of hyperbolic 3manifolds should have some close relationship with another geometric invariant, the Chern-Simons invariant $\operatorname{CS}(M)$. A precise analytic relationship was then conjectured in [30] and proved in [44] (a new proof follows from the work discussed here, see [24]). We will not discuss the definition of this invariant here (it is an invariant of compact riemmanian manifolds, see $[6,5]$, which was extended also to non-compact finite volume hyperbolic 3-manifolds by Meyerhoff [21]). It suffices for the present discussion to know that for a finite volume hyperbolic 3-manifold $M$ the Chern-Simons invariant lies in $\mathbb{R} / \pi^{2} \mathbb{Z}$. Moreover, the combination $\operatorname{vol}(M)+i \operatorname{CS}(M) \in \mathbb{C} / \pi^{2} \mathbb{Z}$ turns out to have good analytic properties and is therefore a natural "complexification" of volume for hyperbolic manifolds. Given this intimate relationship between volume and Chern-Simons invariant, it becomes natural to ask if $C S(M)$ is also detected by $\beta_{0}(M)$. 
The answer, unfortunately, is an easy "no." The point is that $C S(M)$ is an orientation sensitive invariant: $C S(-M)=-C S(M)$, where $-M$ means $M$ with reversed orientation. But, as Gerling pointed out in a letter to Gauss on 15 April 1844: scissors congruence cannot see orientation because any polytope is scissors congruent to its mirror image ${ }^{3}$. Thus $\beta_{0}(-M)=\beta_{0}(M)$ and there is no hope of $C S(M)$ being computable from $\beta_{0}(M)$. This raises the question:

Question 2.2 Is there some way to repair the orientation insensitivity of scissors congruence and thus capture Chern-Simons invariant?

The answer to this question is "yes" and lies in the so called "Bloch group," which was invented for entirely different purposes by Bloch (it was put in final form by Wigner and Suslin). To explain this we start with a result of Dupont and Sah [12] about ideal polytopes - hyperbolic polytopes whose vertices are at infinity (such polytopes exist in hyperbolic geometry, and still have finite volume).

Proposition 2.3 Ideal hyperbolic tetrahedra represent elements in $\mathcal{P}\left(\mathbb{H}^{3}\right)$ and, moreover, $\mathcal{P}\left(\mathbb{H}^{3}\right)$ is generated by ideal tetrahedra.

To help understand this proposition observe that if $A B C D$ is a non-ideal tetrahedron and $E$ is the ideal point at which the extension of edge $A D$ meets infinity then $A B C D$ can be represented as the difference of the two tetrahedra $A B C E$ and $D B C E$, each of which have one ideal vertex. We have thus, in effect, "pushed" one vertex off to infinity. In the same way one can push a second and third vertex off to infinity, ... and the fourth, but this is rather harder. Anyway, we will accept this proposition and discuss its consequence for scissors congruence.

The first consequence is a great gain in convenience: a non-ideal tetrahedron needs six real parameters satisfying complicated inequalities to characterise it up to congruence while an ideal tetrahedron can be neatly characterised by a single complex parameter in the upper half plane.

We shall denote the standard compactification of $\mathbb{H}^{3}$ by $\overline{\mathbb{H}}^{3}=\mathbb{H}^{3} \cup \mathbb{C P}^{1}$. An ideal simplex $\Delta$ with vertices $z_{1}, z_{2}, z_{3}, z_{4} \in \mathbb{C P}^{1}=\mathbb{C} \cup\{\infty\}$ is determined up to congruence by the cross-ratio

$$
z=\left[z_{1}: z_{2}: z_{3}: z_{4}\right]=\frac{\left(z_{3}-z_{2}\right)\left(z_{4}-z_{1}\right)}{\left(z_{3}-z_{1}\right)\left(z_{4}-z_{2}\right)} .
$$

\footnotetext{
${ }^{3}$ Gauss, Werke, Vol. 10, p. 242 ; the argument for a tetrahedron is to barycentrically subdivide by dropping perpendiculars from the circumcenter to each of the faces; the resulting 24 tetrahedra occur in 12 mirror image pairs.
} 
Permuting the vertices by an even (ie orientation preserving) permutation replaces $z$ by one of

$$
z, \quad z^{\prime}=\frac{1}{1-z}, \quad \text { or } \quad z^{\prime \prime}=1-\frac{1}{z} .
$$

The parameter $z$ lies in the upper half plane of $\mathbb{C}$ if the orientation induced by the given ordering of the vertices agrees with the orientation of $\mathbb{H}^{3}$.

There is another way of describing the cross-ratio parameter $z=\left[z_{1}: z_{2}: z_{3}: z_{4}\right]$ of a simplex. The group of orientation preserving isometries of $\mathbb{H}^{3}$ fixing the points $z_{1}$ and $z_{2}$ is isomorphic to the multiplicative group $\mathbb{C}^{*}$ of nonzero complex numbers. The element of this $\mathbb{C}^{*}$ that takes $z_{4}$ to $z_{3}$ is $z$. Thus the cross-ratio parameter $z$ is associated with the edge $z_{1} z_{2}$ of the simplex. The parameter associated in this way with the other two edges $z_{1} z_{4}$ and $z_{1} z_{3}$ out of $z_{1}$ are $z^{\prime}$ and $z^{\prime \prime}$ respectively, while the edges $z_{3} z_{4}, z_{2} z_{3}$, and $z_{2} z_{4}$ have the same parameters $z, z^{\prime}$, and $z^{\prime \prime}$ as their opposite edges. See figure 1 . This description makes clear that the dihedral angles at the edges of the simplex are $\arg (z), \arg \left(z^{\prime}\right), \arg \left(z^{\prime \prime}\right)$ respectively, with opposite edges having the same angle.

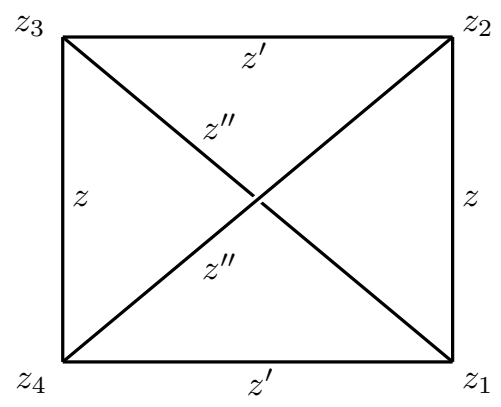

Figure 1

Now suppose we have five points $z_{0}, z_{1}, z_{2}, z_{3}, z_{4} \in \mathbb{C P}^{1}=\mathbb{C} \cup\{\infty\}$. Any fourtuple of these five points spans an ideal simplex, and the convex hull of these five points decomposes in two ways into such simplices, once into two of them and once into three of them. We thus get a scissors congruence relation equating the two simplices with the three simplices. It is often called the "five-term relation." To express it in terms of the cross-ratio parameters it is convenient first to make an orientation convention.

We allow simplices whose vertex ordering does not agree with the orientation of $\mathbb{H}^{3}$ (so the cross-ratio parameter is in the lower complex half-plane) but make the convention that this represents the negative element in scissors congruence. 
An odd permutation of the vertices of a simplex replaces the cross-ratio parameter $z$ by

$$
\frac{1}{z}, \quad \frac{z}{z-1}, \quad \text { or } \quad 1-z
$$

so if we denote by $[z]$ the element in $\mathcal{P}\left(\mathbb{H}^{3}\right)$ represented by an ideal simplex with parameter $z$, then our orientation rules say:

$$
[z]=\left[1-\frac{1}{z}\right]=\left[\frac{1}{1-z}\right]=-\left[\frac{1}{z}\right]=-\left[\frac{z-1}{z}\right]=-[1-z] .
$$

These orientation rules make the five-term scissors congruence relation described above particularly easy to state:

$$
\sum_{i=0}^{4}(-1)^{i}\left[z_{0}: \ldots: \hat{z}_{i}: \ldots: z_{4}\right]=0 .
$$

The cross-ratio parameters occuring in this formula can be expressed in terms of the first two as

$$
\begin{gathered}
{\left[z_{1}: z_{2}: z_{3}: z_{4}\right]=: x \quad\left[z_{0}: z_{2}: z_{3}: z_{4}\right]=: y} \\
{\left[z_{0}: z_{1}: z_{3}: z_{4}\right]=\frac{y}{x} \quad\left[z_{0}: z_{1}: z_{2}: z_{4}\right]=\frac{1-x^{-1}}{1-y^{-1}} \quad\left[z_{0}: z_{1}: z_{2}: z_{3}\right]=\frac{1-x}{1-y}}
\end{gathered}
$$

so the five-term relation can also be written:

$$
[x]-[y]+\left[\frac{y}{x}\right]-\left[\frac{1-x^{-1}}{1-y^{-1}}\right]+\left[\frac{1-x}{1-y}\right]=0 .
$$

We lose nothing if we also allow degenerate ideal simplices whose vertices lie in one plane so the parameter $z$ is real (we always require that the vertices are distinct, so the parameter is in $\mathbb{R}-\{0,1\}$ ), since the five-term relation can be used to express such a "flat" simplex in terms of non-flat ones, and one readily checks no additional relations result. Thus we may take the parameter $z$ of an ideal simplex to lie in $\mathbb{C}-\{0,1\}$ and every such $z$ corresponds to an ideal simplex.

One can show that relations (1) follow from the five-term relation (2), so we consider the quotient

$$
\mathcal{P}(\mathbb{C}):=\mathbb{Z}\langle\mathbb{C}-\{0,1\}\rangle /(\text { five-term relations }(2))
$$

of the free $\mathbb{Z}$-module on $\mathbb{C}-\{0,1\}$. Proposition 2.3 can be restated that there is a natural surjection $\mathcal{P}(\mathbb{C}) \rightarrow \mathcal{P}\left(\mathbb{H}^{3}\right)$. In fact Dupont and Sah (loc. cit.) prove: 
Theorem 2.4 The scissors congruence group $\mathcal{P}\left(\mathbb{H}^{3}\right)$ is the quotient of $\mathcal{P}(\mathbb{C})$ by the relations $[z]=-[\bar{z}]$ which identify each ideal simplex with its mirror image $e^{4}$.

Thus $\mathcal{P}(\mathbb{C})$ is a candidate for the orientation sensitive scissors congruence group that we were seeking. Indeed, it turns out to do (almost) exactly what we want.

The analog of the Dehn invariant has a particularly elegant expression in these terms. First note that the above theorem expresses $\mathcal{P}\left(\mathbb{H}^{3}\right)$ as the "imaginary part" $\mathcal{P}(\mathbb{C})^{-}$(negative co-eigenspace under conjugation ${ }^{5}$ ) of $\mathcal{P}(\mathbb{C})$.

Proposition/Definition 2.5 The Dehn invariant $\delta: \mathcal{P}\left(\mathbb{H}^{3}\right) \rightarrow \mathbb{R} \otimes \mathbb{R} / \pi \mathbb{Q}$ is twice the "imaginary part" of the map

$$
\delta_{\mathbb{C}}: \mathcal{P}(\mathbb{C}) \rightarrow \mathbb{C}^{*} \wedge \mathbb{C}^{*}, \quad[z] \mapsto(1-z) \wedge z
$$

so we shall call this map the "complex Dehn invariant." We denote the kernel of complex Dehn invariant

$$
\mathcal{B}(\mathbb{C}):=\operatorname{Ker}\left(\delta_{\mathbb{C}}\right),
$$

and call it the "Bloch group of $\mathbb{C} . "$

(We shall explain this proposition further in an appendix to this section.)

A hyperbolic 3-manifold $M$ now has an "orientation sensitive scissors congruence class" which lies in this Bloch group and captures both volume and Chern-Simons invariant of $M$. Namely, there is a map

$$
\rho: \mathcal{B}(\mathbb{C}) \rightarrow \mathbb{C} / \pi^{2} \mathbb{Q}
$$

introduced by Bloch and Wigner called the Bloch regulator map, whose imaginary part is the volume map on $\mathcal{B}(\mathbb{C})$, and one has:

Theorem 2.6 ([29], [8]) Let $M$ be a complete oriented hyperbolic 3-manifold of finite volume. Then there is a natural class $\beta(M) \in \mathcal{B}(\mathbb{C})$ associated with $M$ and $\rho(\beta(M))=\frac{1}{i}(\operatorname{vol}(M)+i \mathrm{CS}(M))$.

This theorem answers Question 2.2. But there are still two aesthetic problems:

\footnotetext{
${ }^{4}$ The minus sign in this relation comes from the orientation convention described earlier.

${ }^{5} \mathcal{P}(\mathbb{C})$ turns out to be a $\mathbb{Q}$-vector space and is therefore the sum of its \pm 1 eigenspaces, so "co-eigenspace" is the same as "eigenspace."
} 
- The Bloch regulator $\rho$ plays the rôle for orientation sensitive scissors congruence that volume plays for ordinary scissors congruence. But vol is defined on the whole scissors congruence group $\mathcal{P}\left(\mathbb{H}^{3}\right)$ while $\rho$ is only defined on the kernel $\mathcal{B}(\mathbb{C})$ of complex Dehn invariant.

- The Chern-Simons invariant $\operatorname{CS}(M)$ is an invariant in $\mathbb{R} / \pi^{2} \mathbb{Z}$ but the invariant $\rho(\beta(M))$ only computes it in $\mathbb{R} / \pi^{2} \mathbb{Q}$.

We resolve both these problems in section 4 .

We describe the Bloch regulator map $\rho$ later. It would be a little messy to describe at present, although its imaginary part (volume) has a very nice description in terms of ideal simplices. Indeed, the volume of an ideal simplex with parameter $z$ is $D_{2}(z)$, where $D_{2}$ is the so called "Bloch-Wigner dilogarithm function" given by:

$$
D_{2}(z)=\operatorname{Im}_{2} \ln _{2}(z)+\log |z| \arg (1-z), \quad z \in \mathbb{C}-\{0,1\}
$$

and $\ln _{2}(z)$ is the classical dilogarithm function. It follows that $D_{2}(z)$ satisfies a functional equation corresponding to the five-term relation (see below).

\section{$2.1 \quad$ Further comments}

To worry about the second "aesthetic problem" above could be considered rather greedy. After all, $C S(M)$ takes values in $\mathbb{R} / \pi^{2} \mathbb{Z}$ which is the direct sum of $\mathbb{Q} / \pi^{2} \mathbb{Z}$ and uncountably many copies of $\mathbb{Q}$, and we have only lost part of the former summand. However, it is not even known if the Chern-Simons invariant takes any non-zero values ${ }^{6}$ in $\mathbb{R} / \pi^{2} \mathbb{Q}$. As we shall see, this would be implied by the sufficiency of Dehn invariant for $\mathbb{S}^{3}$ (Conjecture 1.3).

The analogous conjecture in our current situation is:

Conjecture 2.7 Complex Dehn Invariant Sufficiency $\rho: \mathcal{B}(\mathbb{C}) \rightarrow \mathbb{C} / \pi^{2} \mathbb{Q}$ is injective.

Again, the following is known by work of Bloch:

Theorem $2.8 \rho: \mathcal{B}(\mathbb{C}) \rightarrow \mathbb{C} / \pi^{2} \mathbb{Q}$ has countable image.

Thus the complex Dehn invariant sufficiency conjecture would imply:

\footnotetext{
${ }^{6}$ According to J Dupont, Jim Simons deserted mathematics in part because he could not resolve this issue!
} 
Conjecture 2.9 Bloch Rigidity $\mathcal{B}(\mathbb{C})$ is countable.

Theorem $2.10([37,38]) \quad \mathcal{P}(\mathbb{C})$ and its subgroup $\mathcal{B}(\mathbb{C})$ are uniquely divisible groups, so they have the structure of $\mathbb{Q}$-vector spaces. As $\mathbb{Q}$-vector spaces they have infinite rank.

Note that the Bloch group $\mathcal{B}(\mathbb{C})$ is defined purely algebraically in terms of $\mathbb{C}$, so we can define a Bloch group $\mathcal{B}(k)$ analogously ${ }^{7}$ for any field $k$. This group $\mathcal{B}(k)$ is uniquely divisible whenever $k$ contains an algebraically closed field.

It is not hard to see that the rigidity conjecture 2.9 is equivalent to the conjecture that $\mathcal{B}(\overline{\mathbb{Q}}) \rightarrow \mathcal{B}(\mathbb{C})$ is an isomorphism (here $\overline{\mathbb{Q}}$ is the field of algebraic numbers; it is known that $\mathcal{B}(\overline{\mathbb{Q}}) \rightarrow \mathcal{B}(\mathbb{C})$ is injective). Suslin has conjectured more generally that $\mathcal{B}(k) \rightarrow \mathcal{B}(K)$ is an isomorphism if $k$ is the algebraic closure of the prime field in $K$. Conjecture 2.7 has been made in greater generality by Ramakrishnan [32] in the context of algebraic $K$-theory.

Conjectures 2.7 and 2.9 are in fact equivalent to the Dehn invariant sufficiency and rigidity conjectures 1.3 and 1.5 respectively for $\mathbb{H}^{3}$ and $\mathbb{S}^{3}$ together. This is because of the following theorem which connects the various Dehn kernels. It also describes the connections with algebraic $K$-theory and homology of the lie group $\mathrm{SL}(2, \mathbb{C})$ considered as a discrete group. It collates results of of Bloch, Bökstedt, Brun, Dupont, Parry and Sah and Wigner (see [3] and [11]).

Theorem 2.11 There is a natural exact sequence

$$
0 \rightarrow \mathbb{Q} / \mathbb{Z} \rightarrow H_{3}(\mathrm{SL}(2, \mathbb{C})) \rightarrow \mathcal{B}(\mathbb{C}) \rightarrow 0 .
$$

Moreover there are natural isomorphisms:

$H_{3}(\mathrm{SL}(2, \mathbb{C})) \cong K_{3}^{\text {ind }}(\mathbb{C})$,

$H_{3}(\mathrm{SL}(2, \mathbb{C}))^{-} \cong \mathcal{B}(\mathbb{C})^{-} \cong \mathcal{D}\left(\mathbb{H}^{3}\right)$,

$H_{3}(\mathrm{SL}(2, \mathbb{C}))^{+} \cong \mathcal{D}\left(\mathbb{S}^{3}\right) / \mathbb{Z}$ and $\mathcal{B}(\mathbb{C})^{+} \cong \mathcal{D}\left(S^{3}\right) / \mathbb{Q}$,

where $\mathbb{Z} \subset \mathcal{D}\left(S^{3}\right)$ is generated by the class of the 3-sphere and $\mathbb{Q} \subset \mathcal{D}\left(S^{3}\right)$ is the subgroup generated by suspensions of triangles in $\mathbb{S}^{2}$ with rational angles.

The Cheeger-Simons map $c_{2}: H_{3}(\mathrm{SL}(2, \mathbb{C})) \rightarrow \mathbb{C} / 4 \pi^{2} \mathbb{Z}$ of $[5]$ induces on the one hand the Bloch regulator map $\rho: \mathcal{B}(\mathbb{C}) \rightarrow \mathbb{C} / \pi^{2} \mathbb{Q}$ and on the other hand its real and imaginary parts correspond to the volume maps on $\mathcal{D}\left(\mathbb{S}^{3}\right) / \mathbb{Z}$ and $\mathcal{D}\left(\mathbb{H}^{3}\right)$ via the above isomorphisms.

\footnotetext{
${ }^{7}$ Definitions of $\mathcal{B}(k)$ in the literature vary in ways that can mildly affect its torsion if $k$ is not algebraically closed.
} 
The isomorphisms of the theorem are proved via isomorphisms $H_{3}(\mathrm{SL}(2, \mathbb{C}))^{-} \cong$ $H_{3}(\mathrm{SL}(2, R))$ and $H_{3}(\mathrm{SL}(2, \mathbb{C}))^{+} \cong H_{3}(\mathrm{SU}(2))$. We have described the geometry of the isomorphism $\mathcal{B}(\mathbb{C})^{-} \cong \mathcal{D}\left(\mathbb{H}^{3}\right)$ in Theorem 2.4. The geometry of the isomorphism $\mathcal{B}(\mathbb{C})^{+} \cong \mathcal{D}\left(S^{3}\right) / \mathbb{Q}$ remains rather mysterious.

The exact sequence and first isomorphism in the above theorem are valid for any algebraically closed field of characteristic 0 . Thus Conjecture 2.9 is also equivalent to each of the four:

- Is $K_{3}^{\text {ind }}(\overline{\mathbb{Q}}) \rightarrow K_{3}^{\text {ind }}(\mathbb{C})$ an isomorphism? Is $K_{3}^{\text {ind }}(\mathbb{C})$ countable?

- Is $H_{3}(\mathrm{SL}(2, \overline{\mathbb{Q}})) \rightarrow H_{3}(\mathrm{SL}(2, \mathbb{C}))$ an isomorphism? Is $H_{3}(\mathrm{SL}(2, \mathbb{C}))$ countable?

The fact that volume of an ideal simplex is given by the Bloch-Wigner dilogarithm function $D_{2}(z)$ clarifies why the $\mathbb{H}^{3}$ Dehn invariant sufficiency conjecture 1.3 is equivalent to a statement about rational relations among special values of the dilogarithm function. Don Zagier's conjecture about such rational relations, mentioned earlier, is that any rational linear relation among values of $D_{2}$ at algebraic arguments must be a consequence of the relations $D_{2}(z)=D_{2}(\bar{z})$ and the five-term functional relation for $D_{2}$ :

$$
D_{2}(x)-D_{2}(y)+D_{2}\left(\frac{y}{x}\right)-D_{2}\left(\frac{1-x^{-1}}{1-y^{-1}}\right)+D_{2}\left(\frac{1-x}{1-y}\right)=0 .
$$

Differently expressed, he conjectures that the volume map is injective on $\mathcal{P}(\overline{\mathbb{Q}})^{-}$. If one assumes the scissors congruence rigidity conjecture for $\mathbb{H}^{3}$ (that $\mathcal{B}(\overline{\mathbb{Q}})^{-} \cong$ $\mathcal{B}(\mathbb{C})^{-}$) then the Dehn invariant sufficiency conjecture for $\mathbb{H}^{3}$ is just that $D_{2}$ is injective on the subgroup $\mathcal{B}(\overline{\mathbb{Q}})^{-} \subset \mathcal{P}(\overline{\mathbb{Q}})^{-}$, so under this assumption Zagier's conjecture is much stronger. Milnor's conjecture, mentioned earlier, can be formulated that the values of $D_{2}(\xi)$, as $\xi$ runs through the primitive $n$ th roots of unity in the upper half plane, are rationally independent for any $n$. This is equivalent to injectivity modulo torsion of the volume map $D_{2}$ on $\mathcal{B}\left(k_{n}\right)$ for the cyclotomic field $k_{n}=\mathbb{Q}\left(e^{2 \pi i / n}\right)$. For this field $\mathcal{B}\left(k_{n}\right)^{-}=\mathcal{B}\left(k_{n}\right)$ modulo torsion. This is of finite rank but $\mathcal{P}\left(k_{n}\right)^{-}$is of infinite rank, so even when restricted to $k_{n}$ Zagier's conjecture is much stronger than Milnor's. Zagier himself has expressed doubt that Milnor's conjecture can be resolved in the forseeable future.

Conjecture 2.7 can be similarly formulated as a statement about special values of a different dilogarithm function, the "Rogers dilogarithm," which we will define later. 


\subsection{Appendix to section 2: Dehn invariant of ideal polytopes}

To define the Dehn invariant of an ideal polytope we first cut off each ideal vertex by a horoball based at that vertex. We then have a polytope with some horospherical faces but with all edges finite. We now compute the Dehn invariant using the geodesic edges of this truncated polytope (that is, only the edges that come from the original polytope and not those that bound horospherical faces). This is well defined in that it does not depend on the sizes of the horoballs we used to truncate our polytope. (To see this, note that dihedral angles of the edges incident on an ideal vertex sum to a multiple of $\pi$, since they are the angles of the horospherical face created by truncation, which is an euclidean polygon. Changing the size of the horoball used to truncate these edges thus changes the Dehn invariant by a multiple of something of the form $l \otimes \pi$, which is zero in $\mathbb{R} \otimes \mathbb{R} / \pi \mathbb{Q}$.)

Now consider the ideal tetrahedron $\Delta(z)$ with parameter $z$. We may position its vertices at $0,1, \infty, z$. There is a Klein 4 -group of symmetries of this tetrahedron and it is easily verified that it takes the following horoballs to each other:

- At $\infty$ the horoball $\left\{(w, t) \in \mathbb{C} \times \mathbb{R}^{+} \mid t \geq a\right\}$;

- at 0 the horoball of euclidean diameter $|z| / a$;

- at 1 the horoball of euclidean diameter $|1-z| / a$;

- at $z$ the horoball of euclidean diameter $|z(z-1)| / a$.

After truncation, the vertical edges thus have lengths $2 \log a-\log |z|, 2 \log a-$ $\log |1-z|$, and $2 \log a-\log |z(z-1)|$ respectively, and we have earlier said that their angles are $\arg (z), \arg (1 /(1-z)), \arg ((z-1) / z)$ respectively. Thus, adding contributions, we find that these three edges contribute $\log |1-z| \otimes \arg (z)-$ $\log |z| \otimes \arg (1-z)$ to the Dehn invariant. By symmetry the other three edges contribute the same, so the Dehn invariant is:

$$
\delta(\Delta(z))=2(\log |1-z| \otimes \arg (z)-\log |z| \otimes \arg (1-z)) \in \mathbb{R} \otimes \mathbb{R} / \pi \mathbb{Q} .
$$

Proof of Proposition 2.5 To understand the "imaginary part" of $(1-z) \wedge$ $z \in \mathbb{C}^{*} \wedge \mathbb{C}^{*}$ we use the isomorphism

$$
\mathbb{C}^{*} \rightarrow \mathbb{R} \oplus \mathbb{R} / 2 \pi \mathbb{Z}, \quad z \mapsto \log |z| \oplus \arg z,
$$

to represent

$$
\begin{array}{rlr}
\mathbb{C}^{*} \wedge \mathbb{C}^{*} & =(\mathbb{R} \oplus \mathbb{R} / 2 \pi \mathbb{Z}) \wedge(\mathbb{R} \oplus \mathbb{R} / 2 \pi \mathbb{Z}) \\
& =(\mathbb{R} \wedge \mathbb{R}) \oplus(\mathbb{R} / 2 \pi \mathbb{Z} \wedge \mathbb{R} / 2 \pi \mathbb{Z}) \quad \oplus \quad(\mathbb{R} \otimes \mathbb{R} / 2 \pi \mathbb{Z}) \\
& =(\mathbb{R} \wedge \mathbb{R}) \oplus(\mathbb{R} / \pi \mathbb{Q} \wedge \mathbb{R} / \pi \mathbb{Q}) \quad \oplus \quad(\mathbb{R} \otimes \mathbb{R} / \pi \mathbb{Q}),
\end{array}
$$


(the equality on the third line is because tensoring over $\mathbb{Z}$ with a divisible group is effectively the same as tensoring over $\mathbb{Q}$ ). Under this isomorphism we have

$$
\begin{aligned}
&(1-z) \wedge z=(\log |1-z|\wedge \log |z| \oplus \arg (1-z) \wedge \arg z) \\
& \oplus \quad(\log |1-z| \otimes \arg z-\log |z| \otimes \arg (1-z)),
\end{aligned}
$$

confirming the Proposition 2.5.

\section{Computing $\beta(M)$}

The scissors congruence invariant $\beta(M)$ turns out to be a very computable invariant. To explain this we must first describe the "invariant trace field" or "field of definition" of a hyperbolic 3-manifold. Suppose therefore that $M=$ $\mathbb{H}^{3} / \Gamma$ is a hyperbolic manifold, so $\Gamma$ is a discrete subgroup of the orientation preserving isometry group $\operatorname{PSL}(2, \mathbb{C})$ of $\mathbb{H}^{3}$.

Definition 3.1 [33] The invariant trace field of $M$ is the subfield of $\mathbb{C}$ generated over $\mathbb{Q}$ by the squares of traces of elements of $\Gamma$. We will denote it $k(M)$ or $k(\Gamma)$.

This field $k(M)$ is an algebraic number field (finite extension of $\mathbb{Q}$ ) and is a commensurability invariant, that is, it is unchanged on passing to finite covers of $M$ (finite index subgroups of $\Gamma$ ). Moreover, if $M$ is an arithmetic hyperbolic 3manifold (that is, $\Gamma$ is an arithmetic group), then $k(M)$ is the field of definition of this arithmetic group in the usual sense. See $[33,26]$.

Now if $k$ is an algebraic number field then $\mathcal{B}(k)$ is isomorphic to $\mathbb{Z}^{r_{2}} \oplus$ (torsion), where $r_{2}$ is the number of conjugate pairs of complex embeddings $k \rightarrow \mathbb{C}$ of $k$. Indeed, if these complex embeddings are $\sigma_{1}, \ldots, \sigma_{r_{2}}$ then a reinterpretation of a theorem of Borel $[4]$ about $K_{3}(\mathbb{C})$ says:

Theorem 3.2 The "Borel regulator map"

$$
\mathcal{B}(k) \rightarrow \mathbb{R}^{r_{2}}
$$

induced on generators of $\mathcal{P}(k)$ by $[z] \mapsto\left(\operatorname{vol}\left[\sigma_{1}(z)\right], \ldots, \operatorname{vol}\left[\sigma_{r_{2}}(z)\right]\right)$ maps $\mathcal{B}(k) /$ (torsion) isomorphically onto a full lattice in $\mathbb{R}^{r_{2}}$.

A corollary of this theorem is that an embedding $\sigma: k \rightarrow \mathbb{C}$ induces an embedding $\mathcal{B}(k) \otimes \mathbb{Q} \rightarrow \mathcal{B}(C) \otimes \mathbb{Q}$. (This is because the theorem implies that an element of $\mathcal{B}(k)$ is determined modulo torsion by the set of volumes of its Galois 
conjugates, which are invariants defined on $\mathcal{B}(\mathbb{C})$.) Moreover, since $\mathcal{B}(\mathbb{C})$ is a $\mathbb{Q}$-vector space, $\mathcal{B}(\mathbb{C}) \otimes \mathbb{Q}=\mathcal{B}(\mathbb{C})$.

Now if $M$ is a hyperbolic manifold then its invariant trace field $k(M)$ comes embedded in $\mathbb{C}$ so we get an explicit embedding $\mathcal{B}(k(M)) \otimes \mathbb{Q} \rightarrow \mathcal{B}(\mathbb{C})$ whose image, which is isomorphic to $\mathbb{Q}^{r_{2}}$, we denote by $\mathcal{B}(k(M))_{\mathbb{Q}}$.

Theorem $3.3([28,29]) \quad$ The element $\beta(M)$ lies in the subspace $\mathcal{B}(k(M))_{\mathbb{Q}} \subset$ $\mathcal{B}(\mathbb{C})$.

In fact Neumann and Yang show that $\beta(M)$ is well defined in $\mathcal{B}(K)$ for some explicit multi-quadratic field extension $K$ of $k(M)$, which implies that $2^{c} \beta(M)$ is actually well defined in $\mathcal{B}(k(M))$ for some $c$. Moreover, one can always take $c=0$ if $M$ is non-compact, but we do not know if one can for compact $M$.

In view of this theorem we see that the following data effectively determines $\beta(M)$ modulo torsion:

- The invariant trace field $k(M)$.

- The image of $\beta(M)$ in $\mathbb{R}^{r_{2}}$ under the Borel regulator map of Theorem 3.2 .

To compute $\beta(M)$ we need a collection of ideal simplices that triangulates $M$ in some fashion. If $M$ is compact, this clearly cannot be a triangulation in the usual sense. In [29] it is shown that one can use any "degree one ideal triangulation" to compute $\beta(M)$. This means a finite complex formed of ideal hyperbolic simplices plus a map of it to $M$ that takes each ideal simplex locally isometrically to $M$ and is degree one almost everywhere. These always exist (see [29] for a discussion). Special degree one ideal triangulations have been used extensively in practice, eg in Jeff Weeks' program Snappea [43] for computing with hyperbolic 3-manifolds. Oliver Goodman has written a program Snap [17] (building on Snappea) which finds degree one ideal triangulations using exact arithmetic in number fields and computes the invariant trace field and high precision values for the Borel regulator on $\beta(M)$.

Such calculations can provide numerical evidence for the complex Dehn invariant sufficiency conjecture. Here is a typical result of such calculations.

\subsection{Examples}

To ensure that the Bloch group has rank $>1$ we want a field with at least two complex embeddings. One of the simplest is the (unique) quartic field over $\mathbb{Q}$ of 
discriminant 257. This is the field $k=\mathbb{Q}(x) /(f(x))$ with $f(x)=x^{4}+x^{2}-x+1$. This polynomial is irreducible with roots $\tau_{1}^{ \pm}=0.54742 \ldots \pm 0.58565 \ldots i$ and $\tau_{2}^{ \pm}=-0.54742 \ldots \pm 1.12087 \ldots i$. The field $k$ thus has two complex embeddings $\sigma_{1}, \sigma_{2}$ up to complex conjugation, one with image $\sigma_{1}(k)=\mathbb{Q}\left(\tau_{1}^{-}\right)$and one with image $\sigma_{2}(k)=\mathbb{Q}\left(\tau_{2}^{-}\right)$. The Bloch group $\mathcal{B}(k)$ is thus isomorphic to $\mathbb{Z}^{2}$ modulo torsion.

This field occurs as the invariant trace field for two different hyperbolic knot complements in the standard knot tables up to 8 crossings, the 6 -crossing knot $6_{1}$ and the 7 -crossing knot $7_{7}$, but the embeddings in $\mathbb{C}$ are different. For $6_{1}$ one gets $\sigma_{1}(k)$ and for $7_{7}$ one gets $\sigma_{2}(k)$. The scissors congruence classes are

$$
\begin{aligned}
& \beta\left(6_{1}\right)=: \beta_{1}=2\left[\frac{1}{2}\left(1-\tau^{2}-\tau^{3}\right)\right]+[1-\tau]+\left[\frac{1}{2}\left(1-\tau^{2}+\tau^{3}\right)\right] \in \mathcal{B}(k) \\
& \beta\left(7_{7}\right)=: \beta_{2}=4\left[2-\tau-\tau^{3}\right]+4\left[\tau+\tau^{2}+\tau^{3}\right] \in \mathcal{B}(k)
\end{aligned}
$$

where $\tau$ is the class of $x$ in $k=\mathbb{Q}(x) /\left(x^{4}+x^{2}-x+1\right)$. These map under the Borel regulator $\mathcal{B}(k) \rightarrow \mathbb{R}^{2}$ (with respect to the embeddings $\sigma_{1}, \sigma_{2}$ ) to

$6_{1}$ : (3.163963228883143983991014716..,-1.415104897265563340689508587..) $7_{7}$ : (-1.397088165568881439461453224.., 7.643375172359955478221844448..)

In particular, the volumes of these knot complements are 3.1639632288831439.. and 7.6433751723599554.. respectively

Snap has access to a large database of small volume compact manifolds. Searching this database for manifolds whose volumes are small rational linear combinations of $\operatorname{vol}\left(\sigma_{1}\left(\beta_{1}\right)\right)=3.1639632 .$. and $\operatorname{vol}\left(\sigma_{1}\left(\beta_{2}\right)\right)=-1.3970881$.. yielded just eight examples, three with volume $3.16396322888314 .$. , four with volume 4.396672801932495.. and one with volume 5.629382374981847.. . The complex Dehn invariant sufficiency conjecture predicts (under the assumption that the rational dependencies found are exact) that these should all have invariant trace field containing $\sigma_{1}(k)$.

Checking with Snap confirms that their invariant trace fields equal $\sigma_{1}(k)$ and their scissors congruence classes in $\mathcal{B}(k) \otimes \mathbb{Q}$ (computed numerically using the Borel regulator) are $\beta_{1},(3 / 2) \beta_{1}+(1 / 2) \beta_{2}$, and $2 \beta_{1}+\beta_{2}$ respectively.

\section{Extended Bloch group}

In section 2 we saw that $\mathcal{P}(\mathbb{C})$ and $\mathcal{B}(C)$ play a role of "orientation sensitive" scissors congruence group and kernel of Dehn invariant respectively, and that 
the analog of the volume map is then the Borel regulator $\rho$. But, as we described there, this analogy suffers because $\rho$ is defined on the Dehn kernel $\mathcal{B}(\mathbb{C})$ rather than on the whole of $\mathcal{P}(\mathbb{C})$ and moreover, it takes values in $\mathbb{C} / \pi^{2} \mathbb{Q}$, rather than in $\mathbb{C} / \pi^{2} \mathbb{Z}$.

The repair turns out to be to use, instead of $\mathbb{C}-\{0,1\}$, a certain disconnected $\mathbb{Z} \times \mathbb{Z}$ cover of $\mathbb{C}-\{0,1\}$ to define "extended versions" of the groups $\mathcal{P}(\mathbb{C})$ and $\mathcal{B}(\mathbb{C})$. This idea developed out of a suggestion by Jun Yang.

We shall denote the relevant cover of $\mathbb{C}-\{0,1\}$ by $\widehat{\mathbb{C}}$. We start with two descriptions of it. The second will be a geometric interpretation in terms of ideal simplices.

Let $P$ be $\mathbb{C}-\{0,1\}$ split along the rays $(-\infty, 0)$ and $(1, \infty)$. Thus each real number $r$ outside the interval $[0,1]$ occurs twice in $P$, once in the upper half plane of $\mathbb{C}$ and once in the lower half plane of $\mathbb{C}$. We denote these two occurences of $r$ by $r+0 i$ and $r-0 i$. We construct $\widehat{\mathbb{C}}$ as an identification space from $P \times \mathbb{Z} \times \mathbb{Z}$ by identifying

$$
\begin{array}{ll}
(x+0 i, p, q) \sim(x-0 i, p+2, q) & \text { for each } x \in(-\infty, 0) \\
(x+0 i, p, q) \sim(x-0 i, p, q+2) & \text { for each } x \in(1, \infty) .
\end{array}
$$

We will denote the equivalence class of $(z, p, q)$ by $(z ; p, q)$. $\widehat{\mathbb{C}}$ has four components:

$$
\widehat{\mathbb{C}}=X_{00} \cup X_{01} \cup X_{10} \cup X_{11}
$$

where $X_{\epsilon_{0} \epsilon_{1}}$ is the set of $(z ; p, q) \in \widehat{\mathbb{C}}$ with $p \equiv \epsilon_{0}$ and $q \equiv \epsilon_{1}(\bmod 2)$.

We may think of $X_{00}$ as the riemann surface for the function $\mathbb{C}-\{0,1\} \rightarrow \mathbb{C}^{2}$ defined by $z \mapsto(\log z,-\log (1-z))$. If for each $p, q \in \mathbb{Z}$ we take the branch $(\log z+2 p \pi i,-\log (1-z)+2 q \pi i)$ of this function on the portion $P \times\{(2 p, 2 q)\}$ of $X_{00}$ we get an analytic function from $X_{00}$ to $\mathbb{C}^{2}$. In the same way, we may think of $\widehat{\mathbb{C}}$ as the riemann surface for the collection of all branches of the functions $(\log z+p \pi i,-\log (1-z)+q \pi i)$ on $\mathbb{C}-\{0,1\}$.

We can interpret $\widehat{\mathbb{C}}$ in terms of ideal simplices. Suppose we have an ideal simplex $\Delta$ with parameter $z \in \mathbb{C}-\{0,1\}$. Recall that this parameter is associated to an edge of $\Delta$ and that other edges of $\Delta$ have parameters

$$
z^{\prime}=\frac{1}{1-z}, \quad z^{\prime \prime}=1-\frac{1}{z}
$$

with opposite edges of $\Delta$ having the same parameter (see figure 1). Note that $z z^{\prime} z^{\prime \prime}=-1$, so the sum

$$
\log z+\log z^{\prime}+\log z^{\prime \prime}
$$


is an odd multiple of $\pi i$, depending on the branches of log used. In fact, if we use the standard branch of $\log$ then this sum is $\pi i$ or $-\pi i$ depending on whether $z$ is in the upper or lower half plane. This reflects the fact that the three dihedral angles of an ideal simplex sum to $\pi$.

Definition 4.1 We shall call any triple of the form

$$
\mathbf{w}=\left(w_{0}, w_{1}, w_{2}\right)=\left(\log z+p \pi i, \log z^{\prime}+q \pi i, \log z^{\prime \prime}+r \pi i\right)
$$

with

$$
p, q, r \in \mathbb{Z} \quad \text { and } \quad w_{0}+w_{1}+w_{2}=0
$$

a combinatorial flattening for our simplex $\Delta$. Thus a combinatorial flattening is an adjustment of each of the three dihedral angles of $\Delta$ by a multiple of $\pi$ so that the resulting angle sum is zero.

Each edge $E$ of $\Delta$ is assigned one of the components $w_{i}$ of $\mathbf{w}$, with opposite edges being assigned the same component. We call $w_{i}$ the log-parameter for the edge $E$ and denote it $l_{E}(\Delta, \mathbf{w})$.

For $(z ; p, q) \in \widehat{\mathbb{C}}$ we define

$$
\ell(z ; p, q):=(\log z+p \pi i,-\log (1-z)+q \pi i, \log (1-z)-\log z-(p+q) \pi i),
$$

and $\ell$ is then a map of $\widehat{\mathbb{C}}$ to the set of combinatorial flattenings of simplices.

Lemma 4.2 This map $\ell$ is a bijection, so $\widehat{\mathbb{C}}$ may be identified with the set of all combinatorial flattenings of ideal tetrahedra.

Proof We must show that $\left(w_{0}, w_{1}, w_{2}\right)=\ell(z ; p, q)$ determines $(z ; p, q)$. It clearly suffices to recover $z$. But up to sign $z$ equals $e^{w_{0}}$ and $1-z$ equals $e^{-w_{1}}$, and the knowledge of both $z$ and $1-z$ up to sign determines $z$.

\subsection{The extended groups}

We shall define a group $\widehat{\mathcal{P}}(\mathbb{C})$ as $\mathbb{Z}\langle\widehat{\mathbb{C}}\rangle /$ (relations), where the relations in question are a lift of the five-term relations $(2)$ that define $\mathcal{P}(\mathbb{C})$, plus an extra relation that just eliminates an element of order 2 .

We first recall the situation of the five-term relation $(2)$. If $z_{0}, \ldots, z_{4}$ are five distinct points of $\partial \overline{\mathbb{H}}^{3}$, then each choice of four of five points $z_{0}, \ldots, z_{4}$ gives an ideal simplex. We denote the simplex which omits vertex $z_{i}$ by $\Delta_{i}$. We denote 
the cross-ratio parameters of these simplices by $x_{i}=\left[z_{0}: \ldots: \hat{z}_{i}: \ldots: z_{4}\right]$. Recall that $\left(x_{0}, \ldots, x_{4}\right)$ can be written in terms of $x=x_{0}$ and $y=x_{1}$ as

$$
\left(x_{0}, \ldots, x_{4}\right)=\left(x, y, \frac{y}{x}, \frac{1-x^{-1}}{1-y^{-1}}, \frac{1-x}{1-y}\right)
$$

The five-term relation was $\sum_{i=0}^{4}(-1)^{i}\left[x_{i}\right]=0$, so the lifted five-term relation will have the form

$$
\sum_{i=0}^{4}(-1)^{i}\left(x_{i} ; p_{i}, q_{i}\right)=0
$$

with certain relations on the $p_{i}$ and $q_{i}$. We need to describe these relations.

Using the map of Lemma 4.2, each summand in this relation (3) represents a choice $\ell\left(x_{i} ; p_{i}, q_{i}\right)$ of combinatorial flattening for one of the five ideal simplices. For each edge $E$ connecting two of the points $z_{i}$ we get a corresponding linear combination

$$
\sum_{i=0}^{4}(-1)^{i} l_{E}\left(\Delta_{i}, \ell\left(x_{i} ; p_{i}, q_{i}\right)\right)
$$

of log-parameters (Definition 4.1), where we put $l_{E}\left(\Delta_{i}, \ell\left(x_{i} ; p_{i}, q_{i}\right)\right)=0$ if the line $E$ is not an edge of $\Delta_{i}$. This linear combination has just three non-zero terms corresponding to the three simplices that meet at the edge $E$. One easily checks that the real part is zero and the imaginary part can be interpreted (with care about orientations) as the sum of the "adjusted angles" of the three flattened simplices meeting at $E$.

Definition 4.3 We say that the $\left(x_{i} ; p_{i}, q_{i}\right)$ satisfy the flattening condition if each of the above linear combinations (4) of log-parameters is equal to zero. That is, the adjusted angle sum of the three simplices meeting at each edge is zero. In this case relation (3) is an instance of the lifted five-term relation.

There are ten edges in question, so the flattening conditions are ten linear relations on the ten integers $p_{i}, q_{i}$. But these equations turn out to be linearly dependant, and the space of solutions is 5 -dimensional. For example, if the five parameters $x_{0}, \ldots, x_{4}$ are all in the upper half-plane (one can check that this means $y$ is in the upper half-plane and $x$ is inside the triangle with vertices $0,1, y)$ then the conditions are equivalent to:

$$
\begin{gathered}
p_{2}=p_{1}-p_{0}, \quad p_{3}=p_{1}-p_{0}+q_{1}-q_{0}, \quad p_{4}=q_{1}-q_{0} \\
q_{3}=q_{2}-q_{1}, \quad q_{4}=q_{2}-q_{1}-p_{0}
\end{gathered}
$$


which express $p_{2}, p_{3}, p_{4}, q_{3}, q_{4}$ in terms of $p_{0}, p_{1}, q_{0}, q_{1}, q_{2}$. Thus, in this case the lifted five-term relation becomes:

$$
\begin{gathered}
\left(x_{0} ; p_{0}, q_{0}\right)-\left(x_{1} ; p_{1}, q_{1}\right)+\left(x_{2} ; p_{1}-p_{0}, q_{2}\right)- \\
-\left(x_{3} ; p_{1}-p_{0}+q_{1}-q_{0}, q_{2}-q_{1}\right)+\left(x_{4} ; q_{1}-q_{0}, q_{2}-q_{1}-p_{0}\right)=0
\end{gathered}
$$

This situation corresponds to the configuration of figure 2 for the ideal vertices $z_{0}, \ldots, z_{4}$, with $z_{1}$ and $z_{3}$ on opposite sides of the plane of the triangle $z_{0} z_{2} z_{4}$

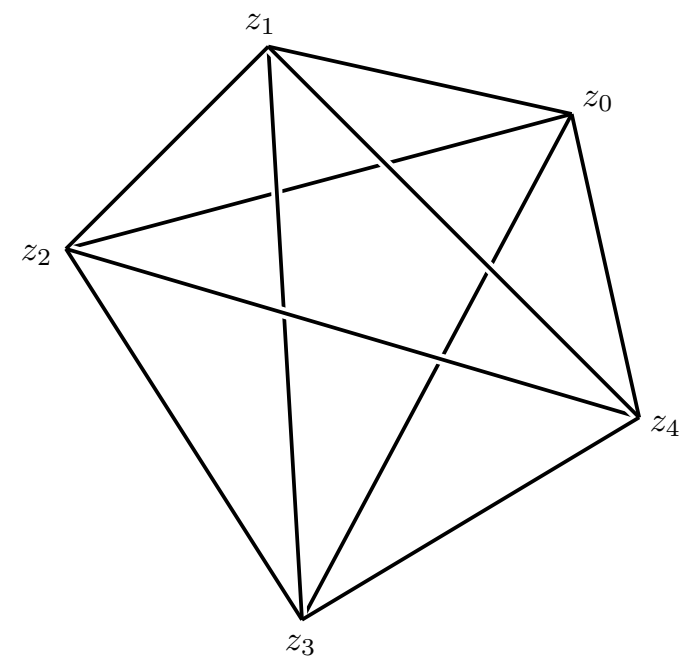

Figure 2

and the line from $z_{1}$ to $z_{3}$ passing through the interior of this triangle.

Definition 4.4 The extended pre-Bloch group $\widehat{\mathcal{P}}(\mathbb{C})$ is the group $\widehat{\mathcal{P}}(\mathbb{C}):=\mathbb{Z}\langle\widehat{\mathbb{C}}\rangle /($ lifted five-term relations and the following relation)

$$
[x ; p, q]+\left[x ; p^{\prime}, q^{\prime}\right]=\left[x ; p, q^{\prime}\right]+\left[x ; p^{\prime}, q\right] .
$$

(We call relation (5) the transfer relation; one can show that if one omits it then $\widehat{\mathcal{P}}(\mathbb{C})$ is replaced by $\widehat{\mathcal{P}}(\mathbb{C}) \oplus \mathbb{Z} / 2$, where the $\mathbb{Z} / 2$ is generated by the element $\kappa:=[x, 1,1]+[x, 0,0]-[x, 1,0]-[x, 0,1]$, which is independant of $x$.)

The relations we are using are remarkably natural. To explain this we need a beautiful version of the dilogarithm function called the Rogers dilogarithm:

$$
\mathcal{R}(z)=-\frac{1}{2}\left(\int_{0}^{z}\left(\frac{\log t}{1-t}+\frac{\log (1-t)}{t}\right) d t\right)-\frac{\pi^{2}}{6} .
$$


The extra $-\pi^{2} / 6$ is not always included in the definition but it improves the functional equation. $\mathcal{R}(z)$ is singular at 0 and 1 and is not well defined on $\mathbb{C}-\{0,1\}$, but it lifts to an analytic function

$$
\begin{gathered}
R: \widehat{\mathbb{C}} \rightarrow \mathbb{C} / \pi^{2} \mathbb{Z} \\
R(z ; p, q)=\mathcal{R}(z)+\frac{\pi i}{2}(p \log (1-z)+q \log z) .
\end{gathered}
$$

We also consider the map

$$
\hat{\delta}: \widehat{\mathbb{C}} \rightarrow \mathbb{C} \wedge \mathbb{C}, \quad \hat{\delta}(z ; p, q)=(\log z+p \pi i) \wedge(-\log (1-z)+q \pi i) .
$$

Relation (5) is clearly a functional equation for both $R$ and $\hat{\delta}$. It turns out that the same is true for the lifted five-term relation. In fact:

Proposition 4.5 If $\left(x_{i} ; p_{i}, q_{i}\right), i=0, \ldots, 4$ satisfy the flattening condition, so

$$
\sum_{i=0}^{4}(-1)^{i}\left(x_{i} ; p_{i}, q_{i}\right)=0
$$

is an instance of the lifted five-term relation, then

$$
\sum_{i=0}^{4}(-1)^{i} R\left(x_{i} ; p_{i}, q_{i}\right)=0
$$

and

$$
\sum_{i=0}^{4}(-1)^{i} \hat{\delta}\left(x_{i} ; p_{i}, q_{i}\right)=0 .
$$

Moreover, each of these equations also characterises the flattening condition.

Thus the flattening condition can be defined either geometrically, as we introduced it, or as the condition that makes the five-term functional equation for either $R$ or $\hat{\delta}$ valid. In any case, we now have:

Theorem 4.6 $R$ and $\hat{\delta}$ define maps

$$
\begin{aligned}
& R: \widehat{\mathcal{P}}(\mathbb{C}) \rightarrow \mathbb{C} / \pi^{2} \mathbb{Z} \\
& \hat{\delta}: \widehat{\mathcal{P}}(\mathbb{C}) \rightarrow \mathbb{C} \wedge \mathbb{C} .
\end{aligned}
$$

We call $\hat{\delta}$ the extended Dehn invariant and call its kernel

$$
\widehat{\mathcal{B}}(\mathbb{C}):=\operatorname{ker}(\hat{\delta})
$$

the extended Bloch group. The final step in our path from Hilbert's 3rd problem to invariants of $3-$ manifolds is given by the following theorem. 
Theorem 4.7 A hyperbolic 3-manifold $M$ has a natural class $\hat{\beta}(M) \in \widehat{\mathcal{B}}(\mathbb{C})$. Moreover, $R(\hat{\beta}(M))=\frac{1}{i}(\operatorname{vol}(M)+i \operatorname{CS}(M)) \in \mathbb{C} / \pi^{2} \mathbb{Z}$.

To define the class $\hat{\beta}(M)$ directly from an ideal triangulation one needs to use a more restrictive type of ideal triangulation than the degree one ideal triangulations that suffice for $\beta(M)$. For instance, the triangulations constructed by Epstein and Penner [15] in the non-compact case and by Thurston [41] in the compact case are of the appropriate type. One then chooses flattenings of the ideal simplices of $K$ so that the whole complex $K$ satisfies certain "flatness" conditions. The sum of the flattened ideal simplices then represents $\hat{\beta}(M)$ up to a $\mathbb{Z} / 6$ correction. The main part of the flatness conditions on $K$ are the conditions that adjusted angles around each edge of $K$ sum to zero together with similar conditions on homology classes at the cusps of $M$. If one just requires these conditions one obtains $\hat{\beta}(M)$ up to 12 -torsion. Additional mod 2 flatness conditions on homology classes determine $\hat{\beta}(M)$ modulo 6 -torsion. The final $\mathbb{Z} / 6$ correction is eliminated by appropriately ordering the vertices of the simplices of $K$. It takes some work to see that all these conditions can be satisfied and that the resulting element of $\widehat{\mathcal{B}}(\mathbb{C})$ is well defined, see $[23,24]$.

\section{Comments and questions}

\subsection{Relation with the non-extended Bloch group}

What really underlies the above Theorem 4.7 is the

Theorem? 5.1 There is a natural short exact sequence

$$
0 \rightarrow \mathbb{Z} / 2 \rightarrow H_{3}(\operatorname{PSL}(2, \mathbb{C}) ; \mathbb{Z}) \rightarrow \widehat{\mathcal{B}}(\mathbb{C}) \rightarrow 0
$$

The reason for the question mark is that, at the time of writing, the proof that the kernel is exactly $\mathbb{Z} / 2$ has not yet been written down carefully.

The relationship of our extended groups with the "classical" ones is as follows. 
Theorem 5.2 There is a commutative diagram with exact rows and columns:

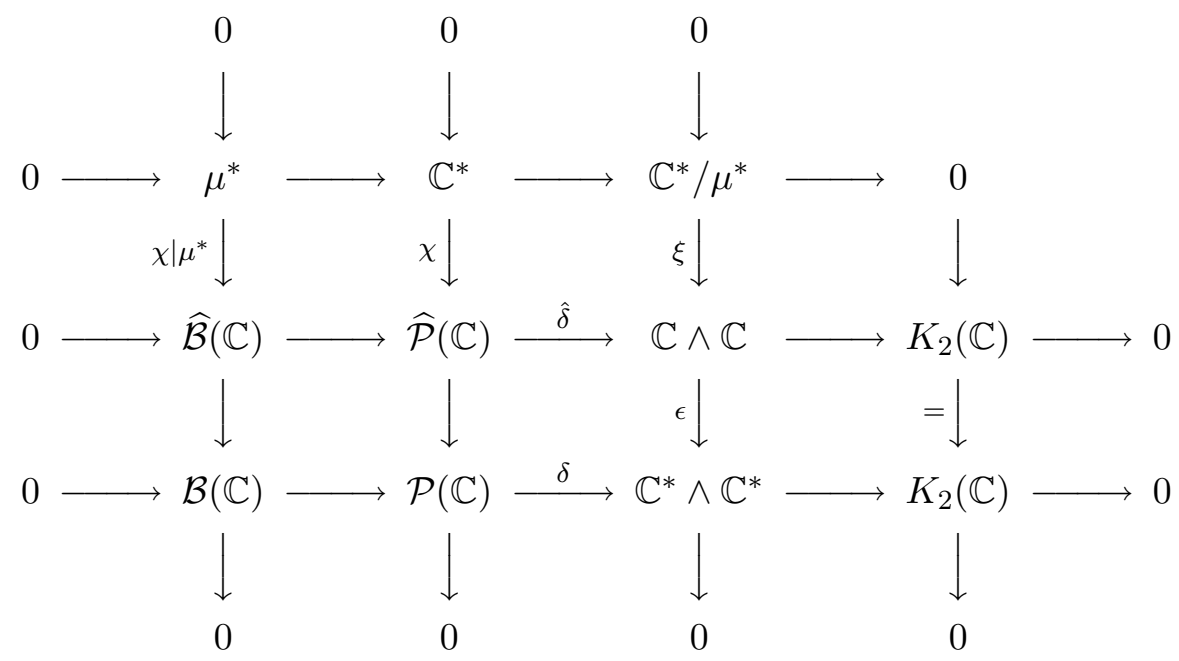

Here $\mu^{*}$ is the group of roots of unity and the labelled maps that have not yet been defined are:

$$
\begin{aligned}
\chi(z) & =[z, 0,1]-[z, 0,0] \in \widehat{\mathcal{P}}(\mathbb{C}) \\
\xi[z] & =\log z \wedge \pi i \\
\epsilon\left(w_{1} \wedge w_{2}\right) & =\left(e^{w_{1}} \wedge e^{w_{2}}\right) .
\end{aligned}
$$

\subsection{Extended extended Bloch}

The use of the disconnected cover $\widehat{\mathbb{C}}$ of $\mathbb{C}-\{0,1\}$ rather than the universal abelian cover (the component $X_{00}$ of $\widehat{\mathbb{C}}$ ) in defining the extended Bloch group may seem unnatural. If one uses $X_{00}$ instead of $\widehat{\mathbb{C}}$ one obtains extended Bloch groups $\mathcal{E P}(\mathbb{C})$ and $\mathcal{E B}(\mathbb{C})$ which are non-trivial $\mathbb{Z} / 2$ extensions of $\widehat{\mathcal{P}}(\mathbb{C})$ and $\widehat{\mathcal{B}}(\mathbb{C})$. Theorem 5.1 then implies a natural isomorphism $H_{3}(\operatorname{PSL}(2, \mathbb{C}) ; \mathbb{Z}) \rightarrow$ $\mathcal{E B}(\mathbb{C})$. The homomorphism of Theorem 5.1 is given explicitely by "flattening" homology classes in the way sketched after Theorem 4.7, and the isomorphism $H_{3}(\mathrm{PSL}(2, \mathbb{C}) ; \mathbb{Z}) \rightarrow \mathcal{E B}(\mathbb{C})$ presumably has a similar explicit description using " $X_{00}$-flattenings," but we have not yet proved that these always exist (note that an $X_{00}$-flattening of a simplex presupposes a choice of a pair of opposite edges of the simplex; changing this choice turns it into a $X_{01}$ - or $X_{10}$-flattening).

For the same reason, we do not yet have a simplicial description of the class $\hat{\beta}(M) \in \mathcal{E} \mathcal{B C}$ for a closed hyperbolic manifold $M$, although this class exists for homological reasons. It is essential here that $M$ be closed - the class 
$\hat{\beta}(M) \in \widehat{\mathcal{B}}(\mathbb{C})$ almost certainly has no natural lift to $\mathcal{E} \mathcal{B}(\mathbb{C})$ in the non-compact case.

The Rogers dilogarithm induces a natural map $R: \mathcal{E B}(\mathbb{C}) \rightarrow \mathbb{C} / 2 \pi^{2} \mathbb{Z}$, and this is the Cheeger-Simons class $H_{3}\left(P S L(2, \mathbb{C}) \rightarrow \mathbb{C} / 2 \pi^{2} \mathbb{Z}\right.$ via the above isomorphism.

\subsection{Computing Chern-Simons invariant}

The formula of [23] for $C S(M)$ used in the programs Snappea and Snap uses ideal triangulations that arise in Dehn surgery. These triangulations are not of the type mentioned after Theorem 4.7 , but by modifying them one can put them in the desired form and use Theorem 4.7 to compute $\hat{\beta}(M)$, reconfirming the formula of $[23]$. The formula computes $C S(M)$ up to a constant for the infinite class of manifolds that arise by Dehn surgery on a given manifold. It was conjectured in [23] that this constant is always a multiple of $\pi^{2} / 6$, and this too is confirmed. The theorem also gives an independent proof of the relation of volume and Chern-Simons invariant conjectured in [30] and proved in [44], from which a formula for eta-invariant was also deduced in [25] and [31].

\subsection{Realizing elements in the Bloch group and Gromov norm}

One way to prove the Bloch group rigidity conjecture 2.9 would be to show that $\mathcal{B}(\mathbb{C})$ is generated by the classes $\beta(M)$ of 3 -manifolds. This question is presumably much harder than the rigidity conjecture, although modifications of it have been used in attempts on it. More specifically, one can ask

Question 5.3 For which number fields $k$ is $\mathcal{B}(k)_{\mathbb{Q}}$ generated as a $\mathbb{Q}$ vector space by classes $\beta(M)$ of 3 -manifolds with invariant trace field contained in $k$ ?

For totally real number fields (ie $r_{2}=0$ ) the answer is trivially "yes" while for number fields with $r_{2}=1$ the existence of arithmetic manifolds again shows the answer is "yes." But beyond this little is known. In fact it is not even known if for every non-real number field $k \subset \mathbb{C}$ a 3 -manifold exists with invariant trace field $k$. (For a few cases, eg multi-quadratic extensions of $\mathbb{Q}$, the author and A Reid have unpublished constructions to show the answer is "yes.")

Jun Yang has pointed out that "Gromov norm" gives an obstruction to a class $\alpha \in \mathcal{B}(\mathbb{C})$ being realizable as $\beta(M)$ (essentially the same observation also occurs in [34]). We define the Gromov norm $\nu(\alpha)$ as

$$
\nu(\alpha)=\inf \left\{\sum\left|\frac{n_{i}}{k}\right|: k \alpha=\sum n_{i}\left[z_{i}\right], \quad z_{i} \in \mathbb{C}\right\},
$$


and it is essentially a result of Gromov, with proof given in [40], that:

\section{Theorem 5.4}

$$
|\operatorname{vol}(\alpha)| \leq V \nu(\alpha),
$$

where $V=1.00149416 \ldots$ is the volume of a regular ideal tetrahedron. If $\alpha=$ $\beta(M)$ for some 3-manifold $M$ then

$$
\operatorname{vol}(\alpha)=V \nu(\alpha) .
$$

In particular, since $\nu(\alpha)$ is invariant under the action of Galois, for $\alpha=\beta(M)$ one sees that the $\operatorname{vol}(M)$ component of the Borel regulator is the largest in absolute value and equals $V \nu(\alpha)$. This suggests the question:

Question 5.5 Is it true for any number field $k$ and for any $\alpha \in \mathcal{B}(k)$ that $V \nu(\alpha)$ equals the largest absolute value of a component of the Borel regulator of $\alpha$ ?

This question is rather naive, and at this point we have no evidence for or against. Another naive question is the following. For $\alpha \in \mathcal{B}(k)_{\mathbb{Q}}$, where $k$ is a number field, we can define a stricter version of Gromov norm by

$$
\nu_{k}(\alpha)=\inf \left\{\sum\left|\frac{n_{i}}{k}\right|: k \alpha=\sum n_{i}\left[z_{i}\right], \quad z_{i} \in k\right\} .
$$

Question 5.6 Is $\nu_{k}(\alpha)=\nu(\alpha)$ for $\alpha \in \mathcal{B}(k)_{\mathbb{Q}}$ ?

If not, then $\nu_{k}$ gives a sharper obstruction to realizing $\alpha$ as $\beta(M)$ since it is easy to show that for $\alpha=\beta(M)$ one has $\operatorname{vol}(\alpha)=V \nu_{K}(\alpha)$ for some at most quadratic extension $K$ of $k$.

\section{References}

[1] C Batut, D Bernardi, H Cohen, M Olivier, Pari-GP, the program, available from: ftp://megrez.ceremab.u-bordeaux.fr/pub/pari/ and http://pari.home.ml.org

[2] S Bloch, Higher regulators, algebraic $K$-theory, and zeta functions of elliptic curves, Lecture notes UC Irvine (1978)

[3] M Bökstedt, M Brun, J L Dupont, Homology of $O(n)$ and $O^{1}(1, n)$ made discrete: an application of edgewise subdivision, J. Pure Appl. Algebra (to appear) 
[4] A Borel, Cohomologie de $S L_{n}$ et valeurs de fonction zeta aux points entiers, Ann. Scuola Norm. Sup. Pisa Cl. Sup. (4) 4 (1977) 613-636

[5] J Cheeger, J Simons, Differential characters and geometric invariants, Springer Lecture Notes in Math. 1167 (1985) 50-80

[6] S Chern, J Simons, Some cohomology classes in principal fiber bundles and their application to Riemannian geometry, Proc. Nat. Acad. Sci. USA 68 (1971) 791-794

[7] M Dehn, Über den Rauminhalt, Math. Ann. 55 (1901) 465-478

[8] J L Dupont, The dilogarithm as a characteristic class for flat bundles, J. Pure and App. Algebra 44 (1987) 137-164

[9] J L Dupont, Algebra of polytopes and homology of flag complexes, Osaka J. Math. 19 (1982) 599-641

[10] J L Dupont, F L Kamber, Cheeger-Chern-Simons classes of transversally symmetric foliations: dependance relations and eta-invariants, Math. Ann. 295 (1993) 449-468

[11] J L Dupont, W Parry, H Sah, Homology of classical Lie groups made discrete II, J. Algebra 113 (1988) 215-260

[12] J L Dupont, H Sah, Scissors congruences II, J. Pure and Appl. Algebra 25 (1982) 159-195

[13] J L Dupont, H Sah, Homology of Euclidean groups of motions made discrete and Euclidean scissors congruences, Acta Math. 164 (1990) 1-27

[14] J L Dupont, H Sah, Three questions about simplices in spherical and hyperbolic 3-space, preprint (1997)

[15] D B A Epstein, R Penner, Euclidean decompositions of non-compact hyperbolic manifolds, J. Diff. Geom. 27 (1988) 67-80

[16] A B Goncharov, Volumes of hyperbolic manifolds and mixed Tate motives, preprint MPI/96-10 Max-Planck-Institut für Math. Bonn (1996)

[17] O Goodman, Snap, the program, (an ARC funded project) available from http://www.ms.unimelb.edu.au/ snap/

[18] D Hilbert, Mathematical Problems, English translation from: "Mathematical developements arising from Hilbert's problems", Proc. Symp. Pure Math. 28 part 1 Amer. Math. Soc. (1976)

[19] B Jessen, The algebra of polyhedra and the Dehn-Sydler theorem, Math. Scand. 22 (1968) 241-256

[20] B Jessen, Zur Algebra der Polytope, Göttinger Nachr. Math. Phys. (1972) 4753

[21] R Meyerhoff, Hyperbolic 3-manifolds with equal volumes but different ChernSimons invariants, from: "Low-dimensional topology and Kleinian groups", D B A Epstein (editor), London Math. Soc. Lecture notes 112 (1986) 209-215 
[22] J Milnor, Hyperbolic geometry: the first 150 years, Bulletin Amer. Math. Soc. 6 (1982) 9-24

[23] W D Neumann, Combinatorics of triangulations and the Chern-Simons invariant for hyperbolic 3-manifolds, from: "Topology 90, Proceedings of the Research Semester in Low Dimensional Topology at Ohio State", Walter de Gruyter Verlag, Berlin-New York (1992) 243-272

[24] W D Neumann, Extended Bloch group and the Chern-Simons class, in preparation

[25] R Meyerhoff, W Neumann, An asymptotic formula for the $\eta$-invariant of hyperbolic 3-manifolds, Comment. Math. Helvetici 67 (1992) 28-46

[26] W D Neumann, A W Reid, Arithmetic of hyperbolic manifolds, from: "Topology 90, Proceedings of the Research Semester in Low Dimensional Topology at Ohio State" Walter de Gruyter Verlag, Berlin-New York (1992) 273-310

[27] W D Neumann, J Yang, Problems for $K$-theory and Chern-Simons Invariants of Hyperbolic 3-Manifolds, L'Enseignement Mathématique 41 (1995) 281296

[28] W D Neumann, J Yang, Invariants from triangulation for hyperbolic 3manifolds, Electronic Research Announcements of the Amer. Math. Soc. 1 (2) (1995) 72-79

[29] W D Neumann, J Yang, Bloch invariants of hyperbolic 3-manifolds, Duke Math. J. (to appear)

[30] W D Neumann, D Zagier, Volumes of hyperbolic 3-manifolds, Topology 24 (1985) 307-332

[31] M Ouyang, A simplicial formula for the eta-invariant of hyperbolic 3manifolds, Topology (to appear)

[32] D Ramakrishnan, Regulators, algebraic cycles, and values of $L$-functions, Contemp. Math. 83 (1989) 183-310

[33] A W Reid, A note on trace-fields of Kleinian groups, Bull. London Math. Soc. 22 (1990) 349-352

[34] A Reznickov, Rationality of secondary classes, J. Diff. Geom. 43 (1996) 674692

[35] C S Sah, Hilbert's Third Problem: Scissors Congruence, Res. Notes in Math. 33, Pitman (1979)

[36] C S Sah, Scissors congruences, I, Gauss-Bonnet map, Math. Scand. 49 (1982) 181-210

[37] A A Suslin, Algebraic K-theory of fields, Proc. Int. Cong. Math. Berkeley 1986, 1 (1987) 222-244

[38] A A Suslin, $K_{3}$ of a field and the Bloch Group, Proc. Steklov Inst. of Math. 183 (1990) English Translation by Amer. Math. Soc. 19914 217-239 
[39] J P Sydler, Conditions nécessaires et sufficiantes pour l'équivalence des polyédres l'espace Euclideien à trois dimensions, Comment. Math. Helv. 42 (1965) 43-80

[40] W P Thurston, The geometry and topology of 3-manifolds, Lecture notes, Princeton University (1977)

[41] W P Thurston, Hyperbolic structures on 3-manifolds I: Deformation of acylindrical manifolds, Annals of Math. 124 (1986) 203-246

[42] W P Thurston, Three dimensional manifolds, Kleinian groups and hyperbolic geometry, Bull. Amer. Math. Soc. 6 (1982) 357-381

[43] J Weeks, Snappea, the program, available from ftp://geom.umn.edu/pub /software/snappea/

[44] T Yoshida, The $\eta$-invariant of hyperbolic 3-manifolds, Invent. Math. 81 (1985) 473-514

[45] D Zagier, The Bloch-Wigner-Ramakrishnan polylogarithm function, Math. Annalen 286 (1990) 613-624

[46] D Zagier, Polylogarithms, Dedekind zeta functions and the algebraic $K$-theory of fields, Arithmetic algebraic geometry, Progr. Math. 89, Birkhäuser Boston, Boston MA, (1991) 391-430

[47] V B Zylev, Equicomposability of equicomplementable polyhedra, Sov. Math. Doklady 161 (1965) 453-455

[48] V B Zylev, $G$-composedness and $G$-complementability, Sov. Math. Doklady 179 (1968) 403-404

Department of Mathematics, The University of Melbourne

Parkville, Vic 3052, Australia

Email: neumann@maths.mu.oz.au

Received: 21 August 1997 